\title{
A Multi-Objective Genetic Algorithm for the Vehicle Routing with Time Windows and Loading Problem
}

\author{
Ana Moura \\ University of Aveiro, \\ Campus Universitário de Santiago, 3810-193 Aveiro, Portugal \\ INESC Coimbra, Institute for Systems and Computers Engeneering, \\ Rua Antero de Quental, nº199, 3000-033 Coimbra, Portugal \\ ana.moura@ua.pt
}

\begin{abstract}
This work presents the Vehicle Routing with Time Windows and Loading Problem (VRTWLP) as a multi-objective optimization problem, implemented within a Genetic Algorithm. Specifically, the three dimensions of the problem to be optimized - the number of vehicles, the total travel distance and volume utilization - are considered to be separated dimensions of a multiobjective space. The quality of the solution obtained using this approach is evaluated and compared with results of other heuristic approaches previously developed by the author. The most significant contribution of this work is our interpretation of VRTWLP as a Multi-objective Optimization Problem.
\end{abstract}

Keywords: Vehicle Routing Problem with Time Windows, Container Loading Problem, Multiobjective Optimization Problem. 